\title{
Cervical trans-spinal direct current stimulation: a modelling-experimental approach
}

Sofia Rita Fernandes ${ }^{1,2^{*}}$ (D), Mariana Pereira ${ }^{1 \dagger}$, Ricardo Salvador ${ }^{3}$, Pedro Cavaleiro Miranda ${ }^{2}$ and Mamede de Carvalho ${ }^{1,4}$

\begin{abstract}
Background: Trans-spinal direct current stimulation (tsDCS) is a non-invasive technique with promising neuromodulatory effects on spinal cord (SC) circuitry. Computational studies are essential to guide effective tsDCS protocols for specific clinical applications. This study aims to combine modelling and experimental studies to determine the electrode montage that maximizes electric field (E-field) delivery during cervical tsDCS.

Methods: Current and E-field distributions in the cervical SC were predicted for four electrode montages in a human realistic model using computational methods. A double-blind crossover and randomized exploratory study was conducted using the montage that maximized E-field delivery. tsDCS was applied for 15 min in 10 healthy subjects (anodal, cathodal, sham, with polarity assigned to the cervical electrode), with a current intensity of $2.5 \mathrm{~mA}$, resulting in a total current charge density delivery of $90 \mathrm{mC} / \mathrm{cm}^{2}$. Upper limb motor (transcranial magnetic stimulation) and sensory evoked potentials (MEP, SEP), M-waves, H-reflex and F-wave responses were analysed. Central and peripheral conduction times were determined using MEP. Repeated measures ANOVA and Friedman test were used for statistical analysis (significance level $a=0.05$ ).

Results: All montages presented higher current density and E-field magnitudes in the cervical SC region between the electrodes. However, electrodes at C3 and T3 spinous processes (C3-T3) originated the highest E-field magnitude $(0.50 \mathrm{~V} / \mathrm{m})$. Using C3-T3 montage we observed significant changes in N9 SEP latency $(p=0.006)$, but significance did not persist in pairwise comparisons (sham-anodal: $p=0.022$; sham-cathodal: $p=0.619$; anodal-cathodal: $p=0.018$; $a=0.017$, Bonferroni corrected). MEP latency and central motor conduction time (CMCT) modified significantly on stimulation ( $p=0.007$ and $p=0.015$, respectively). In addition, pairwise comparisons confirmed significant differences between sham and cathodal conditions after Bonferroni correction for MEP latency (sham-anodal: $p=0.868$; sham-cathodal: $p=0.011$; anodal-cathodal: $p=0.023$ ) and CMCT (sham-anodal: $p=0.929$; sham-cathodal: $p=0.010$; anodalcathodal: $p=0.034$ ).
\end{abstract}

Conclusions: Computational models predicted higher E-field delivery in the cervical SC for the C3-T3 montage. Polarity-dependent effects in motor responses were reported using this montage consistent with spinal motor modulation. tsDCS experimental protocol designs should be guided by modelling studies to improve effectiveness.

Keywords: Direct current stimulation, Spinal cord, Cervical, Neuromodulation, Computational modeling

\footnotetext{
* Correspondence: srcfernandes@fc.ul.pt

Sofia Rita Fernandes and Mariana Pereira are the first two authors provided a

similar contribution for this manuscript

${ }^{1}$ Instituto de Fisiologia, Instituto de Medicina Molecular, Faculdade de

Medicina, Universidade de Lisboa, Avenida Professor Egas Moniz, 1649-028

Lisbon, Portugal

${ }^{2}$ Instituto de Biofísica e Engenharia Biomédica, Faculdade de Ciências,

Universidade de Lisboa, Campo Grande, 1749-016 Lisbon, Portugal

Full list of author information is available at the end of the article
}

(c) The Author(s). 2019 Open Access This article is distributed under the terms of the Creative Commons Attribution 4.0 International License (http://creativecommons.org/licenses/by/4.0/), which permits unrestricted use, distribution, and reproduction in any medium, provided you give appropriate credit to the original author(s) and the source, provide a link to the Creative Commons license, and indicate if changes were made. The Creative Commons Public Domain Dedication waiver (http://creativecommons.org/publicdomain/zero/1.0/) applies to the data made available in this article, unless otherwise stated. 


\section{Background}

Upper limb sensorimotor innervation arises mostly from the cervical region of the spinal cord (SC). Several clinical conditions, such as upper limb weakness, sensory deficit or pain, can be associated with cervical spinal circuitry dysfunctions.

Trans-spinal direct current stimulation (tsDCS) has recently emerged as a non-invasive technique with promising neuromodulatory effects on spinal circuitry related to motor and sensory responses of the upper and lower limbs [7, 8]. tsDCS has a similar approach as transcranial direct current stimulation (tDCS), a noninvasive brain stimulation method for modulating cortical excitability [28]. It applies a constant low intensity electric current through surface electrodes. However, tsDCS and tDCS differ in their principles of application due to the significant heterogeneity of their target tissues. Whereas anodal tDCS produces facilitation in cortical motor responses [28], exploratory tsDCS studies in humans report a variety of polarity-dependent effects in spinal motor responses when stimulating the cervical SC: facilitation of motor responses of abductor digiti minimi (ADM) and abductor policis brevis (APB) muscles was observed during cathodal tsDCS applied over C7 spinous process (s.p.), with the anode over the right deltoid ( $\mathrm{rD}$ ) muscle (C7-rD montage, [4]); increased amplitude of motor evoked potentials (MEP) of the flexor carpi radialis (FCR) was observed independently of the polarity of the electrodes, placed at C7 s.p. and cervicomental angle (C7-CMA montage; [22]). Cervical tsDCS was also observed to increase corticophrenic pathway excitability, considering a C4-CMA montage: increased diaphragmatic MEP amplitude was observed independently of $\mathrm{C} 4$ polarity and tidal volume was increased with the cathode placed at C4 [25].

tsDCS neuromodulatory effects may result from local variations of the current density and electric field (Efield) along neurons, resulting in specific polarizing effects in the transmembrane potential, with axon terminals identified as the dominant cellular target [34, 37]. These variations are affected by various stimulation parameters such as electrode placement and geometry, or injected current intensity and polarity, just as in tDCS $[15,20,41]$.

Computational studies using realistic human models based on MRI are essential tools to predict the electrode montages and stimulation parameters that maximize current delivery and E-field distribution in a specific clinical target $[11,20,24,30]$. There are few modelling studies published on tsDCS delivery on human lumbar and thoracic SC regions; these studies predict maximum E-field magnitude between the electrodes with a stronger longitudinal component in the spinal canal $[15,16,20,31]$. Modelling predictions seem to explain physiological measures obtained during experimental conditions in cervical tsDCS, using MRIbased rat models or simple geometric human models of the cervical SC: diverse electrode montages resulted in different current and E-field distributions, corresponding to different physiological outcomes [12, 49].

The aim of this study is to present a modelling work considering three electrode montages previously explored in the studies cited above, and a longitudinal montage with two electrodes over the SC, since this is thought to result in less variability in experimental results, compared to anterior-posterior montages, as suggested in Dongés et al. [13]. Next, we proceed to present the results of an experimental study carried out in healthy human volunteers using this longitudinal electrode montage, to address neuromodulatory effects on motor and sensory pathways of the upper limb.

\section{Methods \\ Modelling study \\ Human and electrode models design}

A realistic human model was designed based on the 34 years-old male Duke of the Virtual Population Family [9]. Fifteen tissues were considered (Table 1), with no distinction between white matter (WM) and grey matter (GM) in brainstem and cerebellum. The spinal-GM was artificially designed considering SC anatomy [43] and measurements from the Visible Human Data Set (National Library of Medicine, NLM, Visible Human Project ${ }^{\natural}$, www.nlm.nih. gov/research/visible/visible_human.html). The full model was truncated at the level of the thighs and above the elbows, to shorten computational time.

Electrodes were designed as gel and rubber rectangular prism layers, with a metallic rectangular connector on the rubber's surface, considering the Fiab Spa silicone electrode (Vicchio, Italy, http://www.fiab.it) used in the experimental study (Fig. 1a). The three electrodes montages applied in the aforementioned cervical tsDCS studies were modelled first to address the E-field distribution and magnitude variability with electrode position (Fig. 1b): C7-rD [4]; C7-CMA [12, 13, 22]; C4-CMA [25]. Considering the E-field characteristics predicted from the three previous montages, a new electrode placement on C3 and T3 s.p. (C3-T3) was simulated, to test if higher E-fields could be observed in cervical segments related with upper limb function (C4 to T1 spinal segments) compared to $\mathrm{C} 7-\mathrm{rD}$ and CMA montages. C3-T3 was already simulated in a preliminary study from our group, with promising results [14].

Surface meshes were optimized and assembled, and volume meshing was performed with the 3-MATIC module from MIMICS (MIMICS software, v16), resulting in $2.0 \times 10^{7}$ tetrahedral elements for the entire human model with electrodes. 
Table 1 Isotropic electrical conductivities of tissues in the human trunk model

\begin{tabular}{|c|c|c|}
\hline Tissue & $\sigma(S / m)$ & Reference \\
\hline Skin & 0.435 & [17] \\
\hline Fat & 0.040 & [18] \\
\hline Muscle & 0.355 (av) & [39] \\
\hline Lungs & 0.046 (av) & [39] \\
\hline \multirow[t]{2}{*}{ Heart } & 0.535 (av) & [47], \\
\hline & & [18] \\
\hline \multirow[t]{3}{*}{ Viscera (liver, pancreas, stomach, small and large intestine, air) } & 0.123 (av) & [47], \\
\hline & & [29], \\
\hline & & [18] \\
\hline Vertebrae/Bone & 0.006 & [18] \\
\hline Intervertebral disks & 0.200 & [18] \\
\hline Dura mater & 0.030 & [46] \\
\hline CSF & 1.790 & [3] \\
\hline Brainstem / Spinal roots & 0.154 & [18] \\
\hline Cerebellum & 0.290 (av) & [10], [18] \\
\hline Spinal-WM & 0.143 & [18] \\
\hline Spinal-GM & 0.333 & [18] \\
\hline
\end{tabular}

\section{Electrical properties of tissue and electrode materials}

Tissues and materials were assumed to be purely resistive. Isotropic electrical conductivity values were assigned to each tissue in the model after a literature review on DC electrical tissue properties and compiled in Table 1. Cerebellum conductivity resulted from a volumeweighted average of WM and GM conductivities, using volume estimates from Damasceno et al. [10].

Anisotropic conductivity tensors were calculated for muscle and spinal-WM, according to the method described in Fernandes et al. [15], considering different transverse and longitudinal muscle conductivities $\left(\sigma_{\text {trans }}=0.043 \mathrm{~S} / \mathrm{m}\right.$, $\left.\sigma_{\text {long }}=0.667 \mathrm{~S} / \mathrm{m},[39]\right)$, and using the volume constraint from Wolters [48] for the spinal-WM. Conductivity matrices were assigned for each mesh node using a Matlab script (v2015b, www.mathworks.com) and conductivity tensors were interpolated for each volume element using COMSOL Multiphysics (4.3b, www.comsol.com).

A conductivity of $4 \mathrm{~S} / \mathrm{m}$ [23] was assigned to the gel, and rubber pad conductivity was measured to be $44 \pm 1 \mathrm{~S} / \mathrm{m}$, (a)
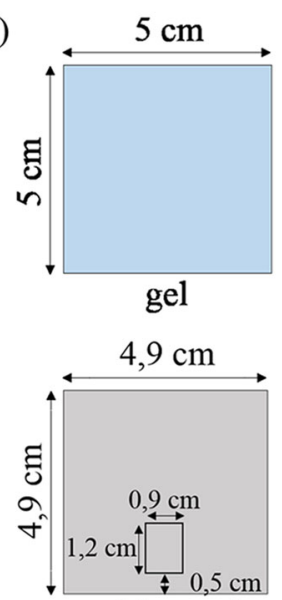

rubber pad (b)
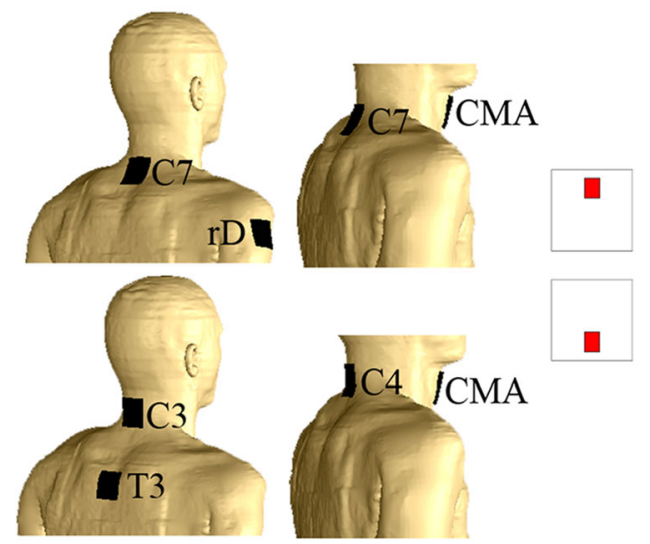

$2,5 \mathrm{~mm} \rightleftharpoons 1 \mathrm{~mm}$

Fig. 1 Electrode settings: (a) gel, rubber pad and connector dimensions; (b) montages considered in the study, with illustration of connector configuration on the right 
according to the method described in Fernandes et al. [15]. Gel and rubber conductivities were considered as isotropic.

\section{Electric field calculation and analysis}

E-field calculations were performed with COMSOL Multiphysics using the finite element method. Current intensity was set to $2.5 \mathrm{~mA}$ and boundary conditions were applied according to Miranda et al. [24], considering electrode connectors as isopotential surfaces. The cervical electrode was considered as the cathode and the other electrode as the anode. Reversing polarity would invert the direction of the E-field but would not affect its magnitude [38]. Four simulations were performed (one per montage), with $2.7 \times 10^{7}$ degrees of freedom and solution time of about 150 min per simulation on a computer with 2 quad-core Intel $^{\circ} \mathrm{Xeon}^{\circ}$ processors clocked at $3.2 \mathrm{GHz}$ and $48 \mathrm{~GB}$ of RAM.

Modelling studies in tDCS reported volume-average Efield values larger than $0.15 \mathrm{~V} / \mathrm{m}$ over the hand knob, when reproducing clinical settings with observed neuromodulatory effects $[24,26,41]$. tsDCS neuromodulation will be assumed if the average E-field exceeds this value in the SC.

E-field components were defined as 3 orthogonal vectors oriented as: caudal-rostral and tangent to SC axis $\left(\vec{E}_{\text {long }}\right)$; ventral-dorsal $\left(\vec{E}_{v d}\right)$; right-left $\left(\vec{E}_{r l}\right)$. Average values in spinal-WM and GM were determined in $1 \mathrm{~mm}$ thick axial slices along the $\mathrm{z}$-axis.

\section{Experimental study}

Higher E-field magnitude in the cervical SC was predicted for C3-T3 montage, therefore we proceeded to a randomized double-blinded observational study in humans to address sensorimotor responses using this montage.

\section{Subjects}

The experimental study was performed on 10 healthy right-handed volunteers (6 women), 22 to 40 years old (mean age 31), recruited from students and staff of the Faculdade de Medicina da Universidade de Lisboa (FMUL). Exclusion criteria applied were: neurologic or psychiatric disease, diabetes, use of biomedical devices. The protocol was approved by the local Ethics Board of the academic centre and all subjects gave informed consent.

\section{Materials and recording conditions}

Skin surface was cleaned with an abrasive gel and a solution of ethyl alcohol $96 \%$ before placing superficial recording and stimulating electrodes. Subjects remained seated in a comfortable armchair. Upper limb was positioned with shoulder in slight abduction $\left(60^{\circ}\right)$, elbow semi-flexed $\left(110^{\circ}\right)$, and forearm pronated and supported by the arm of the chair [33]. The lower limb was also tested to address possible distal effects of the stimulation. It was positioned with $120^{\circ}$ hip flexion, $160^{\circ}$ knee flexion and $110^{\circ}$ plantar flexion of the ankle [33]. The tested muscles were the abductor digiti minimi (ADM) in the right upper limb and the abductor hallux $(\mathrm{AH})$ in the right lower right limb, both were placed in neutral position and relaxed. Each session lasted $90 \mathrm{~min}$ approximately. The temperature of the room was kept constant $\left(23-25^{\circ} \mathrm{C}\right)$.

\section{tsDCS experimental protocol}

DC stimulation was applied by a commercially available stimulator (BrainSTIM $\odot$, SEM, Bologne, Italy) connected to a pair of silicone rubber pad square electrodes $1 \mathrm{~mm}$ thick and $25 \mathrm{~cm}^{2}$ area $\left(5 \times 5 \mathrm{~cm}^{2}\right)$ from Fiab Spa (Vicchio, Italy, www.fiab.it). Electrodes were placed above C3 and T3 s.p.. Conductive gel (Signa $\mathrm{Gel}^{\circ}$, Parker, USA) was applied between the electrodes and the skin to reduce and stabilize contact impedance $(<5 \mathrm{k} \Omega)$ during stimulation [32]. Direct current of $2.5 \mathrm{~mA}$ was applied during $900 \mathrm{~s}$ (30 s ramp-up before tsDCS and $30 \mathrm{~s}$ ramped-down to 0 $\mathrm{mA}$ after tsDCS), with current and charge density of 0.1 $\mathrm{mA} / \mathrm{cm}^{2}$ and $90 \mathrm{mC} / \mathrm{cm}^{2}$, respectively. There are no established safety limits for current density delivery during tsDCS, however, the values indicated above are two orders of magnitude below the threshold limit of $14.3 \mathrm{~mA} / \mathrm{cm}^{2}$ $\left(143 \mathrm{~A} / \mathrm{m}^{2}\right)$ determined by Liebetanz et al. [21], for tDCSinduced brain tissue damage. Also, all subjects tolerated well the experimental setting, with no reported burning or itching sensations. Anodal and cathodal conditions were distinguished only by current polarity, referred to the cervical electrode. Sham tsDCS was delivered during $900 \mathrm{~s}$ with intensity $0 \mathrm{~mA}$, using active ramp stimulation for $30 \mathrm{~s}$ before and after this period, to subjects blind to stimulation condition.

Subjects underwent three sessions of tsDCS, followed by recordings. Session conditions (anodal/cathodal/sham) were randomized with subject and evaluator (MdeC) blinded to the current condition used in each session. Sessions were performed at intervals of at least 1 week in each subject to avoid carry-on effects [27]. Adverse effects, such as burning, itching and pain sensations, were monitored in all subjects.

\section{Sensory responses}

Electrical bipolar stimulation of the right median nerve at wrist was applied to evoke spinal and cortical somatosensory EPs (SEPs). Two sets of 500 stimuli $(0.2 \mathrm{~ms}$ pulse width), with an intensity able to induce a slight muscle contraction were applied at $2 \mathrm{~Hz}$, and recordings were performed with $\mathrm{Ag} / \mathrm{AgCl}$ surface electrodes (impedance $<5 \mathrm{k} \Omega$ ), according to the guidelines for SEPs 
assessment in the upper extremity from the American Clinical Neurophysiology Society (ACNS) [2]. The two sets of recordings were applied to improve SEPs measurements average, as recommended by the ACNS [2]. The recording electrodes were placed on: ipsilateral Erb's point (referred to the contralateral Erb's point); C7 s.p (referred to the anterior neck); $1 \mathrm{~cm}$ behind $\mathrm{C} 3$ and $\mathrm{C} 4$ on the scalp (referred to Fz). Amplitude, peaklatency for N9, N13, N18, N20 and P22 and interpeaklatencies were recorded.

\section{Motor responses}

Neurophysiological motor responses were recorded with EMG equipment (Keypoint $\odot$ Dantec-Natus) using conventional electrodes (Alpine BioMed ${ }^{\circ}$, Denmark, Ref 9013 L0203). Skin impedance under EMG electrodes was kept $<10 \mathrm{k} \Omega$, according to the International Federation of Clinical Neurophysiology guidelines (IFCN [36];).

Motor evoked potentials (MEP) Transcranial magnetic stimulation (TMS) was performed using a conventional circular coil (14 cm diameter coil, Medtronic MagPro@, MagVenture, Denmark), centered on the vertex, to induce maximum electric current near the outer edge of the coil in the motor cortex. Motor responses were recorded from right $\mathrm{ADM}$ and $\mathrm{AH}$ muscles using a belly-tendon montage. Resting motor threshold was estimated as the lowest intensity needed to elicit motor responses $\geq 50 \mu \mathrm{V}$ in at least 5 of 10 stimuli $[35,36]$. MEPs were obtained by stimulating the cortex $20 \%$ above threshold with $30 \mathrm{~s}$ interstimulus interval. We considered mean MEP amplitude and latency from 10 consecutive MEPs. Peripheral and central motor conduction times (PMCT and CMCT, respectively) were calculated for upper limb responses as in Kimura et al. [19]: $\quad \mathrm{PMCT}=1 / 2\left(\mathrm{M}_{\text {latency }}+\mathrm{F}_{\text {minimum }}\right.$ latency -1$)$; $\mathrm{CMCT}=\mathrm{MEP}_{\text {latency }}-\mathrm{PMCT}$.

Cortical silent period (CSP) We stimulated the motor area (stimulus frequency $<0.1 \mathrm{~Hz}$ ) as described above while the subject made a moderate contraction of the right ADM to determine hand CSP (determined from stimulus artefact to electrical signal return with amplitude $>0.1 \mathrm{mV}$ ). We measured the mean CSP duration from 10 sweeps. This method has been shown to be reliable on blind rater evaluation [5].

F-waves and $\mathrm{H}$-reflex responses These responses were measured in 5 subjects to infer lower motor neuron (LMN) excitability following tsDCS. F-waves were recorded from right ADM following supramaximal stimulation of the ulnar nerve at wrist (20 stimuli, $1 \mathrm{~Hz}$, filter setting $20-10,000 \mathrm{~Hz}$ ). We evaluated mean peak-to-peak amplitude, negative-peak area, minimum, maximum and mean latency, chronodispersion and persistence before and after each intervention.

H-reflex was recorded using a bipolar montage over the flexor carpi radialis (FCR) muscle, and by delivering $1 \mathrm{~ms}$ rectangular stimuli in the median nerve at elbow. The frequency of stimulation was $0.5 \mathrm{~Hz}$ to avoid postactivation depression [33]. Applied current intensity was progressively increased to obtain $\mathrm{H}$-reflex in steps of 0.2 $\mathrm{mA}$, until eliciting the maximal M-wave. H-reflex threshold, maximum H-reflex amplitude and minimum latency, and $\mathrm{H}: \mathrm{M}$ ratio were evaluated.

\section{Statistical analysis}

Experimental sessions were compared considering the factor condition (sham, cathodal, anodal) using repeated-measures ANOVA. The Greenhouse-Geisser correction was applied when Mauchly's test of sphericity was significant. The Friedman test (with Wilcoxon test for paired comparisons) was applied for non-normal data. A $p$-value $<0.05$ was set as significant following Bonferroni correction for multiple comparisons. Data is presented as mean \pm standard deviation (STD). Calculations were performed with IBM SPSS, version 25.

\section{Results \\ Modelling study}

This section presents the main results for current density and E-field distribution in the spinal cord and adjacent regions. Additional information can be found in Additional file 1.

\section{Current density distribution in the human model}

Current flows longitudinally from anode to cathode in the spinal canal, due to anatomical shape and high CSF conductivity, decreasing from skin to spinal-GM by two orders of magnitude (Fig. 2). Current orientation changes in C4-CMA at the level of the edge of CMA active connector (C6/C7 vertebral region; Fig. 2d, inset). This may result from the combination of anatomical characteristics, such as different electrical conductivities, and electrode position. Cerebellum and brainstem present local current maxima, which are larger in montages with electrodes placed at a higher cervical position (C4-CMA; C3-T3). These local maxima may be originated by current flow originated in the highly conductive muscle and CSF tissues.

\section{E-field distribution in the spinal cord and adjacent regions}

Figure 3 presents profiles of the E-field magnitude and of its components averaged over the spinal-WM along the caudal-rostral direction. Similar profiles were observed for the spinal-GM. Considering $0.15 \mathrm{~V} / \mathrm{m}$ as the minimum E-field value for neuromodulation, different montages address different clinical targets, as shown in 


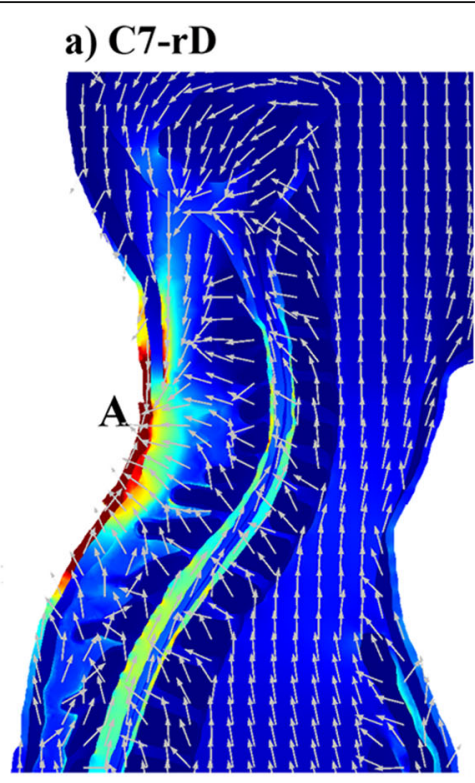

c) $\mathrm{C3}-\mathrm{T} 3$

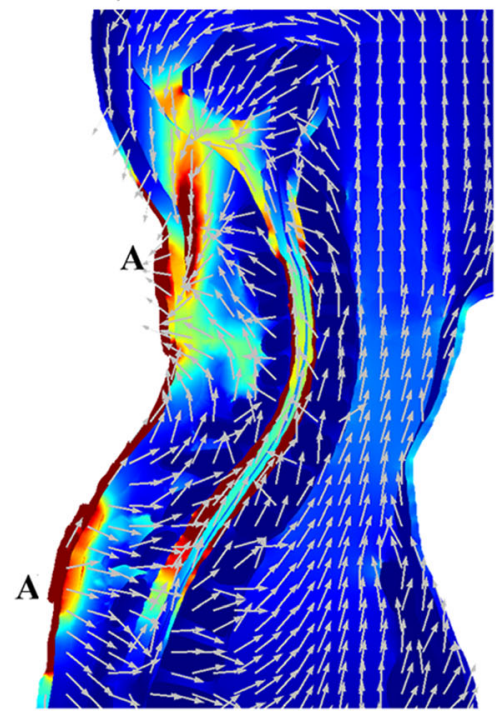

b) C7-CMA



d) C4-CMA
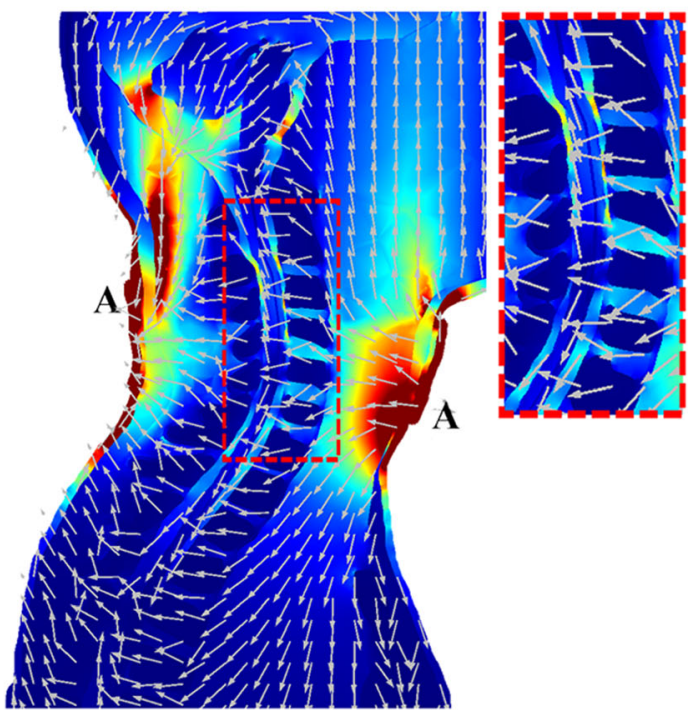

Fig. 2 Current density magnitude distribution in a sagittal slice of the upper thoracic and neck regions for each electrode montage, with the current direction represented by grey arrows of the same length: a) C7-rD, b) C7-CMA; c) C3-T3; d) C4-CMA. Letter "A" marks the active connector position in each electrode. The magnitude colour scale is on the top right and is the same for all montages. The inset on the right of C4-CMA shows the reversal in current density direction inside the spinal canal

Table 2. All montages may modulate upper limb functions, higher cervical montages (C4-CMA, C3-T3) may also address mechanical-related respiratory functions. C3-T3 presents the highest magnitudes, reaching 0.40 and $0.50 \mathrm{~V} / \mathrm{m}$ in C6-C7 segments of the spinal-GM and WM, respectively. $\mathrm{E}_{\text {long }}$ makes the largest contribution to the E-field magnitude in C7-rD and C3-T3, as $\mathrm{E}_{\mathrm{vd}}$ and $E_{r l}$ have much lower values, reflecting the longitudinal nature of the field. CMA montages have comparable $E_{\text {long }}$ and $E_{v d}$ components in the spinal-WM, due to the posterior-anterior electrodes location. $\mathrm{E}_{\mathrm{long}}$ reverses direction along the SC in C4-CMA as in the current density profile (Fig. 3, lower right).

The E-field value also exceeds $0.15 \mathrm{~V} / \mathrm{m}$ in posterior regions of the brainstem and cerebellum, especially in higher cervical montages (C3-T3 and C4-CMA), which is consistent with the current density magnitude observed in these regions (Fig. $2 \mathrm{~b}$ and $\mathrm{c}$ ); this may indicate that neuromodulation of these regions can also occur during tsDCS application.

E-field localized maxima or hotspots appear in the same regions of the spinal-GM and WM in all montages. 


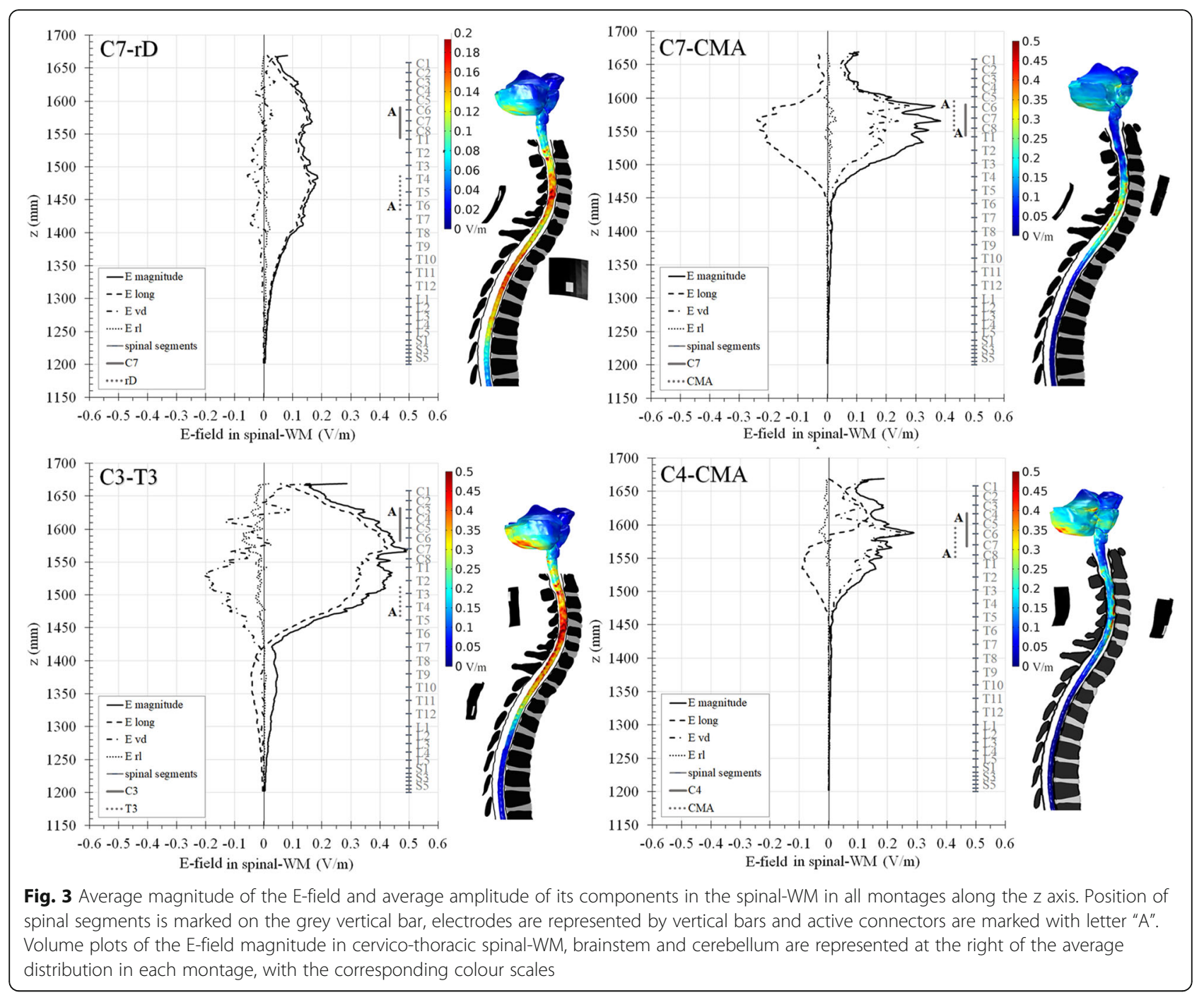

Moderate to strong coefficients of determination were found for inverse function fits between E-field magnitude $\left(\mathrm{E}_{\mathrm{mag}}\right)$ and CSF volume $\left(\mathrm{V}_{\mathrm{CSF}}\right)$ distributions in local spinal regions for each montage $\left(\mathrm{V}_{\mathrm{CSF}} \times \mathrm{E}_{\text {mag }}{ }^{\mathrm{a}}=\right.$ constant $\left.(a>0), R^{2}=0.5-0.9\right)$. CSF narrowing in the spinal canal may be the main anatomical feature causing E-field hotspots. However, disks and vertebrae bony edges protrusions may cause CSF narrowing and originate hotspots indirectly.

Axial slices of the E-field magnitude in spinal segments near maxima present an almost constant magnitude in the
GM, with local maxima near WM/GM interface, especially at dorsal and ventral horns, where most collateral fibres enter or leave the GM (Fig. 4). C3 and C7 segments have different maxima positions in spinal-WM when comparing CMA montages with C7-rD and C3-T3: local maxima appear also in the anterior regions in CMA montages. This indicates an influence on electrode position: the CMA montage originates a higher ventraldorsal component that originates higher E-field in the anterior regions of the spinal-WM segments near or between the electrodes.

Table 2 Spinal segments with E-field magnitude above $0.15 \mathrm{~V} / \mathrm{m}$ and related body regions

\begin{tabular}{lllll}
\hline Montages & C7-rD & C7-CMA & C4-CMA & C3-T3 \\
\hline Spinal-WM & C6-C7, T2-T5 & C4-T1 & C2-T1 & C1-T5 \\
Spinal-GM & C6-C7, T2-T5 & C5-T1 & - & C1-T4 \\
Body regions & Upper limb & Neck, Shoulder, & Neck, Diaphragm, Shoulder, & Neck, Diaphragm, Shoulder, \\
& Upper thoracic region & Upper limb, Wrist, Hand & Upper limb, Wrist, Hand & $\begin{array}{l}\text { Upper limb, Wrist, Hand Upper } \\
\text { thoracic region }\end{array}$ \\
\hline
\end{tabular}




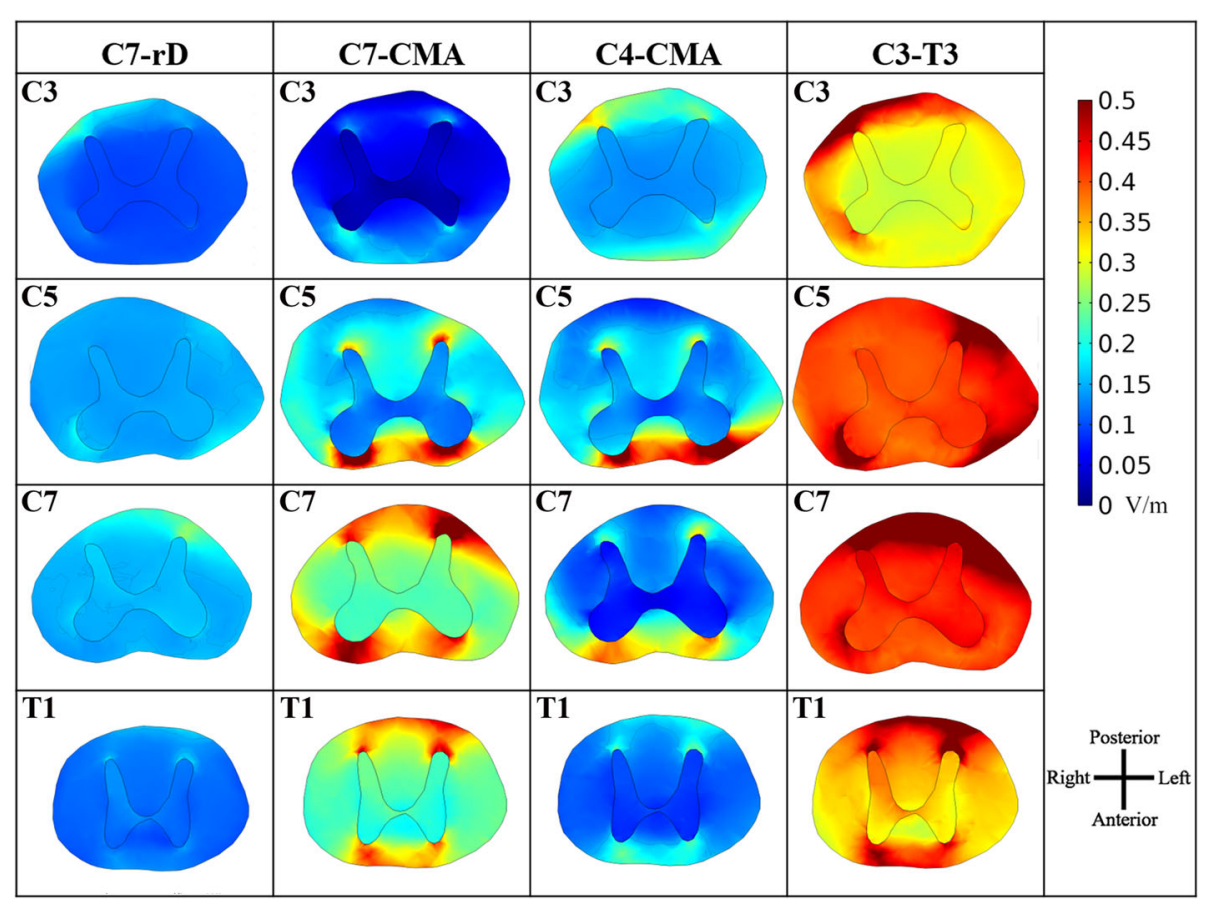

Fig. 4 Axial SC slices in selected cervical and T1 segments near maximum E-field peaks. Colour scale and image orientation are represented on the right

\section{Experimental study}

Only the most significant changes are reported in this section. Additional information on experimental measurements, statistics and comparison tests results is reported in Additional file 1.

\section{Sensory pathways}

N9, N13, N18, N20 and P22 amplitudes and peak and interpeak latencies were measured immediately after each stimulation condition (sham, cathodal, anodal). SEPs amplitudes and interpeak latencies did not present statistically significant differences. SEPs mean peak latencies presented a tendency to increase after anodal tsDCS (Fig. 5). N9 SEP values attained statistical significance in multiple comparisons between conditions $(\mathrm{F}(2,18)=6.797, p=0.006)$, however this difference was not maintained after pairwise comparisons (sham-anodal: $p=0.022$; sham-cathodal: $p=0.619$; anodal-cathodal: $p=0.018$; Bonferroni corrected: $\alpha=$ $0.05 / 3=0.017$; Fig. 5). No polarity-dependent neuromodulation on upper limb sensory responses can be ascertained from the results presented.

\section{Motor pathways}

CSP duration and MEP responses (amplitude, area and latency) were measured in the upper limb after sham, anodal and cathodal conditions (Fig. 6). MEP latency recorded in ADM was the only measurement with a significant change $(\mathrm{F}(2,18)=3.139, p=0.007$, Table 3$)$. Bonferroni corrected pairwise comparisons revealed a statistically significant difference between cathodal and sham conditions (sham-anodal: $p=0.868$; sham-cathodal: $p=0.011$; anodal-cathodal: $p=0.023)$, with cathodal tsDCS resulting in a shorter mean latency. There were no significant changes from multiple comparisons for MEP amplitude $(\mathrm{F}(2,18)=1.303, p=0.296)$, MEP area $(\mathrm{F}(2$, $18)=1.324, p=0.291)$ and CSP duration $(\mathrm{F}(2,18)=0.184$, $p=0.834$; Table 3). Distal effects of tsDCS were addressed with lower limb MEP responses, however these did not present significant changes in amplitude, area and latency $(p>0.05$; Table 3).

$\mathrm{M}$-wave, F-wave and H-reflex responses were measured in 5 subjects to address if MEP latency changes could be due to central or peripheral effects of stimulation. There were no statistically significant changes in multiple comparisons between sham, anodal and cathodal conditions for $\mathrm{M}$-wave, $\mathrm{H}$-reflex, $\mathrm{H}$ :M ratio and F-wave measurements, as reported in Table $3(p>0.05$ in all comparisons). Combining the results from F-wave and MEP responses, the PMCT and CMCT were determined in the 5 subjects. Only the CMCT presented statistically significant differences in multiple comparisons $(\mathrm{F}(2,8)=7.422, p=0.015$, Table 3$)$, a significant reduction was disclosed following cathodal stimulation (sham-anodal: $p=0.929$; sham-cathodal: $p=0.010$; anodal-cathodal: $p=$ 0.034, $\alpha=0.017$, Bonferroni corrected, Fig. 6). 

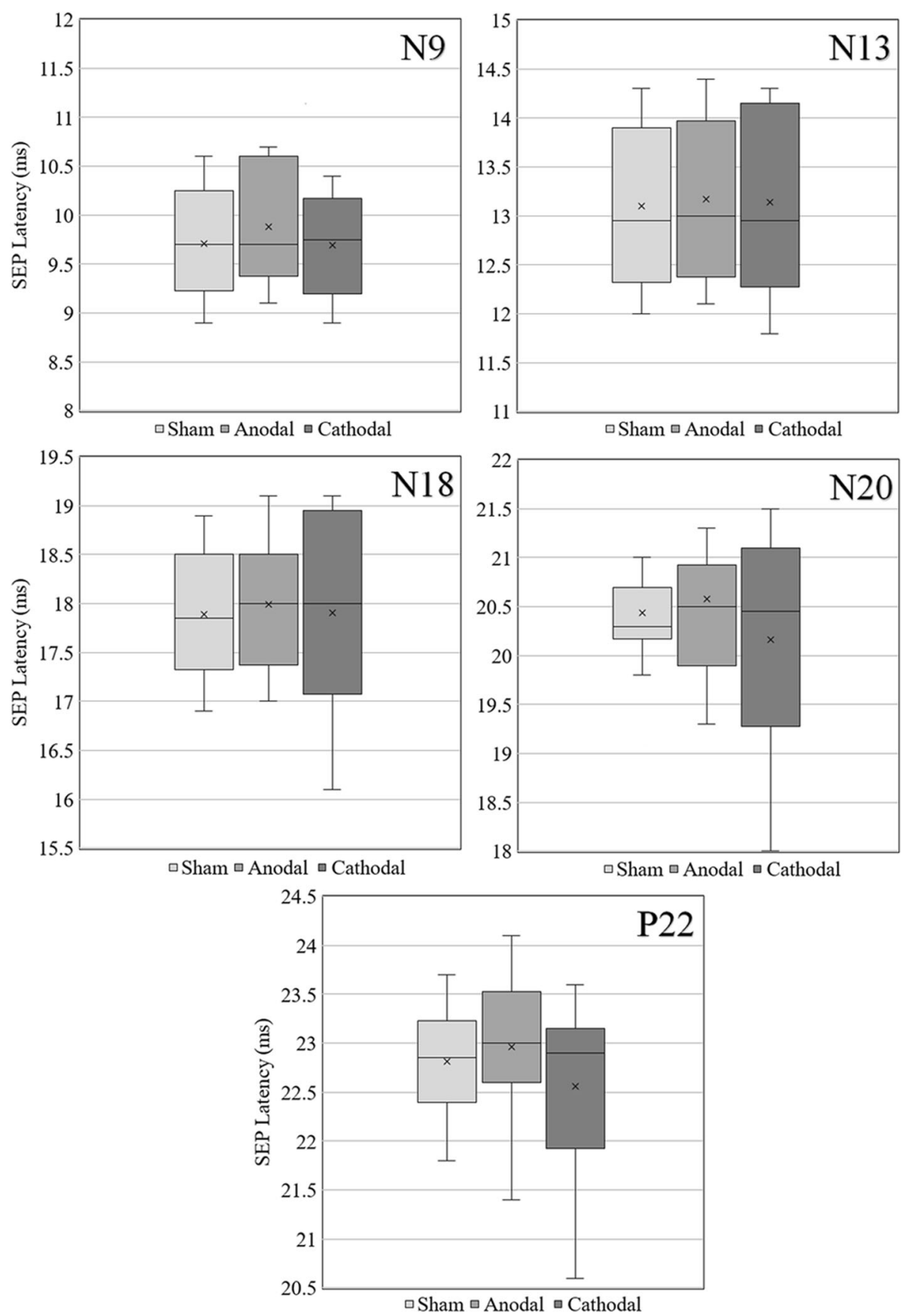

Fig. 5 Boxplots of N9, N13, N18, N20 and P22 SEP's latency for sham, anodal and cathodal conditions

\section{Discussion}

\section{Current density and E-field predictions from the modelling study}

Current density and E-field distributions have a higher longitudinal component in the SC. Longitudinal fields were reported in other modelling studies on thoracic and lumbar tsDCS $[15,16,20,31]$, This longitudinal tendency is due to the cable-like structure of the spine, with a conductive core (spinal cord and CSF) surrounded by an insulating sheath (vertebral column).

All montages present regions with E-field magnitude above $0.15 \mathrm{~V} / \mathrm{m}$, which is in line with the observed neuromodulatory effects using C7-rD, C7-CMA and C4-CMA, however these regions are located in different part of the SC (Fig. 3, Table 2), which may account for the differences observed between studies using different montages. Niérat 


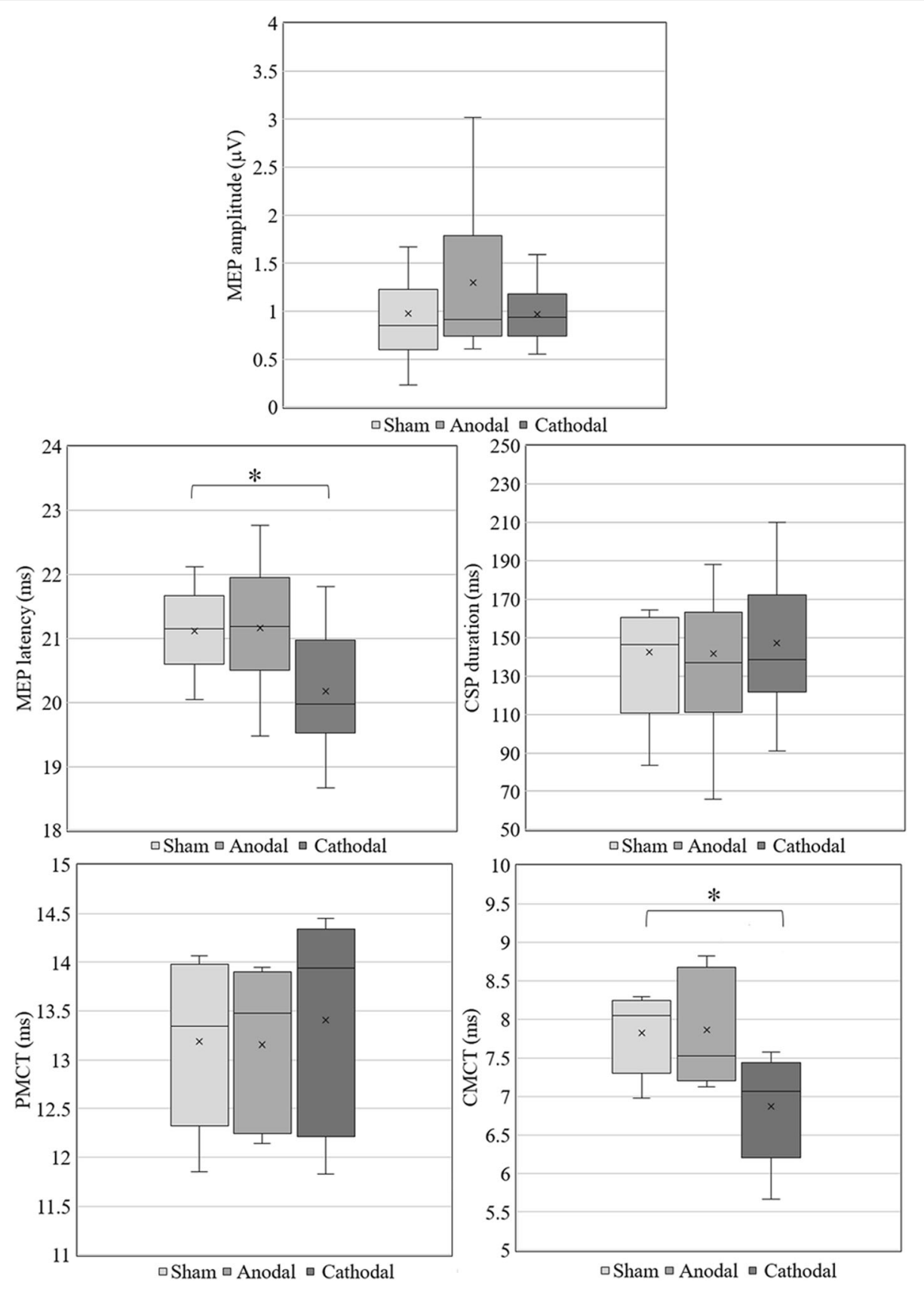

Fig. 6 Boxplots of upper limb MEP amplitude and latency, CSP duration, PMCT and CMCT for sham, anodal and cathodal conditions. Statistically significant differences between conditions (sham-cathodal) are marked by * $(p<0.05 / 3$, Bonferroni corrected) in MEP latency and CMCT

et al. [25] observed increased excitability of the corticophrenic pathway using C4-CMA montage, which is consistent with a larger E-field magnitude predicted in C3-C5 segments, related with the phrenic nerve (Fig. 3). Bocci et al. [4] reported improved upper limb motor recruitment and shortening of peripheral silent period (PSP) after cathodaltsDCS using C7-rD, which is predicted to induce a stronger field in C6-C7 spinal segments, from where part of the brachial plexus arises. Exploratory studies using C7-CMA montage report different effects, such as MEP amplitude increase without changes in H-reflex [22] or only acute changes in MEP amplitude during combined cathodaltsDCS and cervicomedullary stimulation [12]. The length of E-field maximum regions also indicates which montages are suitable for wider (C3-T3, C7-rD) or more focal (CMA montages) stimulation.

Local E-field maxima appear mostly near the WM/GM interface at the dorsal and ventral horns (Fig. 4). Previous 
Table 3 Neurophysiological motor responses for upper and lower limb (ADM: abductor digiti minimi muscle; AH: abductor hallux muscle; CMCT: central motor conduction time; CSP: cortical silent period; MEP: motor evoked potential; PMCT: peripheral motor conduction time; SEP: somatosensory evoked potential)

\begin{tabular}{|c|c|c|c|c|c|c|c|c|}
\hline \multicolumn{2}{|c|}{ Neurophysiological parameters } & \multicolumn{3}{|c|}{ Mean \pm STD } & \multicolumn{4}{|c|}{ repeated-measures ANOVA results } \\
\hline & & \multirow{2}{*}{$\frac{\text { Sham }}{1.0 \pm 0.6}$} & \multirow{2}{*}{$\frac{\text { Anodal }}{1.3 \pm 0.8}$} & \multirow{2}{*}{$\begin{array}{l}\text { Cathodal } \\
1.0 \pm 0.3\end{array}$} & \multirow{2}{*}{$\frac{F}{1.303}$} & \multirow{2}{*}{$\frac{d f}{2}$} & \multirow{2}{*}{$\frac{\mathrm{df} \text { error }}{18}$} & \multirow{2}{*}{$\frac{p \text { value }}{0.296}$} \\
\hline upper limb MEP (ADM) & Amplitude $(\mu \mathrm{V})$ & & & & & & & \\
\hline & Area $(\mathrm{mA})$ & $1.6 \pm 1.4$ & $2.1 \pm 1.4$ & $1.5 \pm 0.6$ & 1.324 & 2 & 18 & 0.291 \\
\hline & Latency (ms) & $21.1 \pm 0.6$ & $21.1 \pm 1.0$ & $20.2 \pm 1.0$ & 3.139 & 2 & 18 & $0.007^{a}$ \\
\hline \multirow[t]{3}{*}{ lower limb MEP (AH) } & Amplitude $(\mu \mathrm{V})$ & $1.4 \pm 0.9$ & $1.4 \pm 0.7$ & $1.4 \pm 0.8$ & 0.009 & 2 & 18 & 0.991 \\
\hline & Area $(m A)$ & $2.5 \pm 2.1$ & $2.5 \pm 1.5$ & $2.4 \pm 1.7$ & 0.025 & 2 & 18 & 0.976 \\
\hline & Latency (ms) & $37.5 \pm 2.2$ & $38.2 \pm 2.3$ & $37.6 \pm 2.1$ & 1.184 & 2 & 18 & 0.329 \\
\hline CSP & Duration (ms) & $143 \pm 43$ & $142 \pm 49$ & $147 \pm 36$ & 0.184 & 2 & 18 & 0.834 \\
\hline M-wave & Amplitude (mV) & $4.5 \pm 1.7$ & $4.7 \pm 2.2$ & $3.8 \pm 1.7$ & 1.998 & 2 & 8 & 0.198 \\
\hline (median) & Latency (ms) & $3.1 \pm 0.2$ & $3.1 \pm 0.4$ & $3.2 \pm 0.3$ & 0.201 & 2 & 8 & 0.822 \\
\hline \multirow[t]{4}{*}{ H-reflex (median) } & $\mathrm{H}_{\max }(\mathrm{mV})$ & $0.3 \pm 0.2$ & $0.3 \pm 0.2$ & $0.5 \pm 0.6$ & 1.104 & 2 & 8 & 0.377 \\
\hline & Min Lat (ms) & $21.2 \pm 1.6$ & $21.0 \pm 2.1$ & $22.3 \pm 2.6$ & 1.284 & 2 & 8 & 0.328 \\
\hline & $\mathrm{H}: \mathrm{M}$ & $0.08 \pm 0.05$ & $0.06 \pm 0.03$ & $0.12 \pm 0.10$ & 1.601 & 2 & 8 & 0.260 \\
\hline & Threshold (mA) & $3.4 \pm 1.4$ & $3.8 \pm 1.1$ & $3.5 \pm 1.3$ & 0.582 & 2 & 8 & 0.581 \\
\hline M-wave & Amplitude (mV) & $16.4 \pm 3.3$ & $16.1 \pm 3.6$ & $16.0 \pm 3.3$ & 0.562 & 2 & 8 & 0.591 \\
\hline (ulnar) & Latency (ms) & $2.3 \pm 0.1$ & $2.2 \pm 0.2$ & $2.4 \pm 0.3$ & 4.072 & 2 & 8 & 0.060 \\
\hline \multirow[t]{7}{*}{ F-wave (ulnar) } & Amplitude $(\mu \mathrm{V})$ & $206 \pm 163$ & $203 \pm 155$ & $214 \pm 145$ & 0.310 & 2 & 8 & 0.742 \\
\hline & Area $(\mu \mathrm{A})$ & $903 \pm 838$ & $794 \pm 761$ & $890 \pm 683$ & 0.924 & 2 & 8 & 0.436 \\
\hline & Min Lat (ms) & $25.1 \pm 1.8$ & $25.1 \pm 1.7$ & $25.4 \pm 2.3$ & 0.454 & 2 & 8 & 0.651 \\
\hline & Mean Lat (ms) & $26.8 \pm 1.8$ & $27.2 \pm 1.9$ & $27.6 \pm 1.6$ & 1.530 & 2 & 8 & 0.274 \\
\hline & Max Lat (ms) & $29.0 \pm 1.9$ & $29.1 \pm 2.0$ & $29.8 \pm 2.3$ & 1.046 & 2 & 8 & 0.395 \\
\hline & Chronodispersion $^{c}$ & $3.9 \pm 1.4$ & $4.0 \pm 1.3$ & $4.4 \pm 1.7$ & $0.508^{b}$ & 1.041 & 4.164 & 0.521 \\
\hline & Persistence & $18 \pm 2$ & $16 \pm 5$ & $18 \pm 3$ & 2.344 & 2 & 8 & 0.158 \\
\hline $\mathrm{PMCT}^{\mathrm{d}}$ & Duration (ms) & $13.2 \pm 0.9$ & $13.2 \pm 0.9$ & $13.4 \pm 1.1$ & 1.106 & 2 & 8 & 0.377 \\
\hline \multirow[t]{2}{*}{$\mathrm{CMCT}^{\mathrm{e}}$} & Duration (ms) & $7.8 \pm 0.5$ & $7.9 \pm 0.8$ & $6.9 \pm 0.7$ & 7.422 & 2 & 8 & $0.015^{\mathrm{a}}$ \\
\hline & Min Duration (ms) & $7.1 \pm 0.7$ & $6.7 \pm 0.9$ & $6.3 \pm 1.0$ & 2.610 & 2 & 8 & 0.134 \\
\hline
\end{tabular}

Statistically significant differences within subjects $(p<0.05)$

${ }^{\mathrm{b}}$ Greenhouse-Geisser correction

${ }^{c}$ Chronodispersion $(\mathrm{ms})=$ maximum latency - minimum latency

${ }^{\mathrm{d}} \mathrm{PMCT}=0.5 \times$ (M-wave mean latency $+\mathrm{F}$-wave minimum latency - 1)

${ }^{\mathrm{e}} \mathrm{CMCT}=0.5 \times(\mathrm{MEP}$ mean latency $-\mathrm{PMCT}) ; \mathrm{CMCT}$ minimum $=0.5 \times(\mathrm{MEP}$ minimum latency $-\mathrm{PMCT})$

numerical modelling studies on invasive spinal cord stimulation found that the EF component parallel to fibres (corresponding to $\mathrm{E}_{\text {long }}$ in $\mathrm{SC}$ ) have an influence on the transmembrane potential of collateral fibres, originating from the spinal-WM columns, as they bent into the spinalGM [45]. The same effect was also predicted in transcranial magnetic stimulation (TMS): there may be stimulation of pyramidal tract neurons in the regions where these fibres bend after entering the cortical WM [40]. The neuromodulatory effects that were observed in the exploratory studies on cervical tsDCS mentioned above may be due to the strong E-field variations along the collateral fibres as they bend and pass the WM/GM interface.

The E-field reaches magnitudes above $0.15 \mathrm{~V} / \mathrm{m}$ in posterior regions of brainstem and cerebellum in higher cervical electrode montages (C4-CMA, C3-T3), thus neuromodulation of vegetative functions can be considered (Fig. 3). Since these tissues were considered homogeneous, a model with distinct WM and GM will allow more accurate predictions of tsDCS effects.

The inverse relations found between E-field magnitude and CSF volume distributions were also observed in thoracic and lumbar tsDCS studies [15, 16]. Individual anatomical variability may originate different tsDCS clinical outcomes, thus subject-specific models should be considered to predict the optimal electrode montages.

\section{Neuromodulatory effects of C3-T3 montage}

C3-T3 resulted in the highest current density and E-field magnitudes, with maxima at C6-C7 spinal segments, 
where part of the upper limb innervation arises. Longitudinal bipolar montages (both electrodes over the SC) or monopolar montages (one electrode over the target area and the other at a considerable distance) were recommended by Dongés et al. [13] for upper limb function neuromodulation, due to the strong longitudinal component induced. We observed neuromodulatory effects on upper limb sensorimotor responses using C3-T3.

\section{Effects on sensory responses}

A significant difference was only detected for SEP N9 $(\mathrm{F}(2,18)=6.797, p=0.006)$, however the significance did not resist pairwise comparisons (Fig. 5). Brachial plexus EP/N9 SEP responses are mainly generated by nerve trunk and roots activity close to the SC [6]. Our model contains nerve exits through vertebral foramina with poor anatomical detail. The E-field distributions presented hotspots near foramina, as seen in animal modelling studies [42]. Spinal roots may contribute to dorsal root ganglia and peripheral nerve excitation, due to a local current focusing caused by the CSF high conductivity near vertebral foramina, supporting our observation.

\section{Effects on motor responses}

MEP latency in ADM decreased significantly by cathodal stimulation compared with sham condition (Fig. 6). This indicates a polarity-dependent facilitation of motor responses with the cathodal condition, probably caused by increased LMN excitability. In addition, we observed a statistically significant decrease in CMCT by cathodal stimulation, with no changes in PCMT. Both findings are in agreement with previous observations, where a cathodal-dependent LMN facilitation is reported (e.g. [4, 25]). Struijk et al. [44] predicted that cervical epidural stimulation polarity effects vary with spinal dorsal fibres orientation with respect to the Efield: longitudinal and tangential dorsal fibres depolarize near the cathode and radial fibres near the anode. The high $\mathrm{E}_{\text {long }}$ component predicted in C3-T3 may originate the effects observed on MEP latency, not reported previously in other studies. CSP duration was also addressed in the study to observe if there were effects on inhibitory pathways; however, we did not disclose significant differences. In addition, no effect was observed in lower limb MEP responses (Table 3), suggesting that tsDCS neuromodulation may have only a local effect, without evidence of propagated supra or infraspinal levels effect.

M-waves, H-reflex, F-waves and conduction times were assessed in 5 subjects to address possible tsDCS effects on LMN excitability, but the results were not conclusive. We observed a non-significant increase of the $\mathrm{H}: \mathrm{M}$ ratio mean values after cathodal condition (Table 3), which might indicate a facilitated MN recruitment by Ia stimulation, also consistent with an increased spinal excitability. One limitation is the small number of tested subjects, in the future this should be repeated in a larger population.

\section{Considerations on methodology and future research}

The number of tissues included in the model was a trade-off between accuracy in field estimates and highquality volume meshing, to avoid excessive computation time and memory costs. The artificially designed spinal-GM mask is a low-resolution contour that does not represent an accurate change in size across the cervical enlargement. Even so, it was relevant for a more realistic E-field prediction, due to the different WM and GM electric properties.

Future modelling work should address spinal networks to predict the influence of the E-field in neuronal transmembrane potential and understand the variability of observations. Considering the influence of CSF narrowing predicted by the model, it will be important to determine the influence of inter-subject variability in tsDCS outcomes by comparing E-field predictions in different human models.

One of the main limitations of this study is the sample size formed by young subjects. A larger sample with wider age range would be advisable to confirm our experimental findings reported. Also, recording responses during tsDCS and after tsDCS offset could inform on acute and after effects, as observed previously (e.g. [1, 12, 13, 26, 27]). Two further limitations of our study is that we have not explored the modulation of corpus callosum by testing ipsilateral CSP and we did not evaluate phrenic nerve responses to test neuromodulation of C3-5 motor nuclei. Future studies should also address neuromodulation of cerebellum and brainstem circuitry using rostral cervical montages, considering the values of the predicted E-field in those regions.

\section{Conclusion}

This study presents evidence of neuromodulatory effects on sensorimotor spinal pathways using a new cervical tsDCS montage, informed by a computational study based on a realistic human model. Since anatomical features and electrode position influence current and E-field profiles, future tsDCS experimental studies should be optimized by computational models to design effective tsDCS protocols.

\section{Supplementary information}

Supplementary information accompanies this paper at https://doi.org/10. 1186/s12984-019-0589-6.

\footnotetext{
Additional file 1. This file presents plots on the average E-field magnitude in the spinal-GM segments and volume distribution of the E-field magnitude in brainstem and cerebellum, results on correlation fits performed to investigate the influence of anatomical features in local E-field magnitude maxima, and tables summarizing the sensory responses registered during the cervical tsDCS protocol.
} 


\section{Abbreviations}

ACNS: American Clinical Neurophysiology Society; ADM: Abductor digiti minimi muscle; AH: Abductor hallux muscle; ANOVA: Analysis of variance; APB: Abductor policis brevis muscle; CMA: Cervicomental angle: CMCT: Central motor conduction time; CSF: Cerebrospinal fluid; CSP: Cortical silent period; DC: Direct current; E, E-field: Electric field;

EMG: Electromyography; FCR: Flexor carpi radialis; FCT: Fundação para a Ciência e Tecnologia; FMUL: Faculdade de Medicina da Universidade de Lisboa; GM: Grey matter; IFCN: International Federation of Clinical Neurophysiology; long: longitudinal; MCTES: Ministério da Ciência, Tecnologia e Ensino Superior; MEP: Motor evoked potential; MN: Motor neuron; MRI: Magnetic resonance imaging; NLM: National Library of Medicine; PMCT: Peripheral motor conduction time; rD: right deltoid; rl: rightleft; s.p.: spinous process; SC: spinal cord; SEP: Somatosensory evoked potential; STD: Standard deviation; tDCS: transcranial direct current stimulation; TMS: Transcranial magnetic stimulation; tsDCS: trans-spinal direct current stimulation; vd: ventral-dorsal; WM: white matter

\section{Acknowledgements}

Not applicable.

\section{Authors' contributions}

SRF and PCM conceived the modelling study; SRF, MP and MdeC conceived the experimental study; SRF performed the computational modelling calculations; RS calculated the spinal-WM conductivity tensor; MP collected all experimental data; SRF analysed all experimental data; SRF and MP drafted the paper; RS, PCM and MdeC offered critical revisions; all authors reviewed, read and approved the final manuscript.

\section{Funding}

All the experimental protocol expenses related with design of the study, collection of data and publication fees were supported by UID/BIM/50005/ 2019, project funded by Fundação para a Ciência e a Tecnologia (FCT)/ Ministério da Ciência, Tecnologia e Ensino Superior (MCTES) through Fundos do Orçamento de Estado. All expenses related with computational modelling and analysis of data were supported by the FCT in the scope of the FCT-IBEB Strategic Project UID/BIO/00645/2013. S. R. Fernandes was supported by a FCT PhD grant, reference SFRH/BD/100254/2014.

\section{Availability of data and materials}

The datasets used and/or analysed during the current study are available from the corresponding author on reasonable request.

\section{Ethics approval and consent to participate}

The experimental procedure was designed and performed in accordance with the relevant guidelines and regulations, particularly those set out in the Declaration of Helsinki pertaining to the ethical treatment of human subjects. Participants signed informed consents, and were instructed on their rights as participants, including the right to withdraw from the experiment at any time without fear of negative consequences. The study protocol has been approved by the Ethics Committee of the Centro Hospitalar Lisboa Norte and Centro Académico de Medicina de Lisboa (approval code number 327/17).

\section{Consent for publication}

Not applicable.

\section{Competing interests}

PC Miranda is a member of the advisory board of Neuroelectrics, Barcelona, Spain.

\section{Author details}

${ }^{1}$ Instituto de Fisiologia, Instituto de Medicina Molecular, Faculdade de Medicina, Universidade de Lisboa, Avenida Professor Egas Moniz, 1649-028 Lisbon, Portugal. ${ }^{2}$ Instituto de Biofísica e Engenharia Biomédica, Faculdade de Ciências, Universidade de Lisboa, Campo Grande, 1749-016 Lisbon, Portugal. ${ }^{3}$ Neuroelectrics, Avinguda Tibidabo, 47 bis, 08035 Barcelona, Spain. ${ }^{4}$ Departamento de Neurociências e Saúde Mental, Hospital de Santa Maria Centro Hospitalar Lisboa Norte, Avenida Professor Egas Moniz, 1649-035 Lisbon, Portugal.
Received: 14 May 2019 Accepted: 6 September 2019

Published online: 25 October 2019

\section{References}

1. Ahmed Z. Trans-spinal direct current stimulation alters muscle tone in mice with and without spinal cord injury with spasticity. J Neurosci. 2014;34(5):1701-9.

2. American Clinical Neurophysiology Society (ACNS). Guideline 9D: guidelines on short-latency somatosensory evoked potentials. J Clin Neurophysiol. 2006;23(2):168-79.

3. Baumann SB, Wozny D, Kelly S, Meno F. The electrical conductivity of human cerebrospinal fluid at body temperature. IEEE Trans Biomed Eng. 1997:44(3):220-3.

4. Bocci T, Vanninia B, Torzini A, Mazzatenta A, Vergari M, Cogiamanian F, et al. Cathodal transcutaneous spinal direct current stimulation (tsDCS) improves motor unit recruitment in healthy subjects. Neurosci Lett. 2014;578:75-9.

5. Cerqueira V, De Mendonça A, Minez A, Dias AR, De Carvalho M. Does caffeine modify corticomotor excitability? Neurophysiol Clin. 2006;36(4): 219-26.

6. Chiappa K. Evoked potentials in clinical medicine. 3rd ed. Philadelphia: Lippincott-Raven Publishers; 1997.

7. Cogiamanian F, Vergari M, Pulecchi F, Marceglia S, Priori A. Effect of spinal transcutaneous direct current stimulation on somatosensory evoked potentials in humans. Clin Neurophysiol. 2008;119:2636-40.

8. Cogiamanian F, Ardolino G, Vergari M, Ferrucci R, Ciocca M, Scelzo E, et al. Transcutaneous spinal direct current stimulation. Front Psychiatry. 2012;3:63.

9. Christ A, Kainz W, Hahn EG, Honegger K, Zefferer M, Neufeld E, et al. The virtual family-development of surface based anatomical models of two adults and two children for dosimetric simulations. Phys Med Biol. 2010;55: N23-38.

10. Damasceno A, Damasceno BP, Cendes F. The clinical impact of cerebellar Grey matter pathology in multiple sclerosis. PLoS One. 2014;9(5):e96193.

11. Datta A, Bansal V, Diaz J, Patel J, Reato D, Bikson M. Gyri-precise head model of transcranial direct current stimulation: improved spatial focality using a ring electrode versus conventional rectangular pad. Brain Stimul. 2009;2(4):201-7.

12. Dongés SC, Bai S, Taylor JL. Concurrent electrical cervicomedullary stimulation and cervical transcutaneous spinal direct current stimulation result in a stimulus interaction. Exp Physiol. 2017a;102(10):1309-20.

13. Dongés SC, D'Amico JM, Butler JE, Taylor JL. The effects of cervical transcutaneous spinal direct current stimulation on motor pathways supplying the upper limb in humans. PLoS One. 2017b;12(2):e0172333.

14. Fernandes SR, Salvador R, Wenger $C$, de Carvalho MA, Miranda PC. Influence of electrode configuration on the electric field distribution during transcutaneous spinal direct current stimulation of the cervical spine. Conf Proc IEEE Eng Med Biol Soc. 2016;2016:3121-4. https://doi.org/10.1109/ EMBC.2016.7591390.

15. Fernandes SR, Salvador R, Wenger $C$, de Carvalho M, Miranda PC. Transcutaneous spinal direct current stimulation of the lumbar and sacral spinal cord: a modelling study. J Neural Eng. 2018;15(3):036008.

16. Fiocchi S, Ravazzani P, Priori A, Parazzini M. Cerebellar and spinal direct current stimulation in children: computational modeling of the induced electric field. Front Hum Neurosci. 2016;10:522.

17. Geddes LA, Baker LE. The specific resistance of biological materials - a compendium of data for the biomedical engineer and physiologist. Med Bio Eng. 1967:5(3):271-93.

18. Haueisen J, Ramon C, Eiselt M, Brauer H, Nowak $H$. Influence of tissue resistivities on neuromagnetic fields and electric potentials studied with a finite element model of the head. IEEE Trans Biomed Eng. 1997;44(8): 727-35.

19. Kimura J. Electrodiagnosis in diseases of nerve and muscle: principles and practise. 3d ed. New York: Oxford University Press; 2001.

20. Kuck A, Stegeman D, van Asseldonk E. Modeling trans-spinal direct current stimulation for the modulation of the lumbar spinal motor pathways. J Neural Eng. 2017;14(5):056014

21. Liebetanz D, Koch R, Mayenfels S, Konig F, Paulus W, Nitsche MA. Safety limits of cathodal transcranial direct current stimulation in rats. Clin Neurophysiol. 2009;120:1161-7.

22. Lim CY, Shin HI. Noninvasive DC stimulation on neck changes MEP. Neuroreport. 2011;22(16):819-23. 
23. Minhas P, Bansal V, Patel J, Ho JS, Diaz J, Datta A, Bikson M. Electrodes for high-definition transcutaneous DC stimulation for applications in drugdelivery and electrotherapy, including tDCS. J Neurosci Methods. 2010; 190(2):188-97.

24. Miranda PC, Mekonnen A, Salvador R, Ruffini G. The electric field in the cortex during transcranial current stimulation. Neuroimage. 2013;70:48-58.

25. Niérat M, Similowski T, Lamy J. Does trans-spinal direct current stimulation Alter phrenic Motoneurons and respiratory Neuromechanical outputs in humans? A double-blind, sham-controlled, randomized, crossover study. J Neurosci. 2014;34(43):14420-9.

26. Nitsche MA, Paulus W. Excitability changes induced in the human motor cortex by weak transcranial direct current stimulation. J Physiol. 2000;527(3):633-9.

27. Nitsche MA, Liebetanz D, Lang N, Antal A, Tergau F, Paulus W, Priori A. Safety criteria for transcranial direct current stimulation (tDCS) in humans. Clin Neurophysiol. 2003;114(11):2220-3.

28. Nitsche MA, Cohen LG, Wasserman EM, Priori A, Lang N, Antal A, et al. Transcranial direct current stimulation: state of the art 2008. Brain Stimul. 2008;1(3):206-23.

29. Osswald K. Measurement of the conductivity and dielectric constants of biological tissues and liquids by microwave. Hochfrequentz Tech. Elektroakustik. 1937;49:40-9.

30. Opitz A, Paulus W, Will S, Antunes A, Thielscher A. Determinants of the electric field during transcranial direct current stimulation. Neuroimage. 2015;109:140-50. https://doi.org/10.1016/j.neuroimage.2015.01.033.

31. Parazzini M, Fiocchi S, Liorni I, Rossi E, Cogiamanian F, Vergari M, et al. Modelling the current density generated by transcutaneous spinal direct current stimulation (tsDCS). Clin Neurophysiol. 2014;125(11):2260-70.

32. Peterchev AV, Wagner TA, Miranda PC, Nitsche MA, Paulus W, Lisanby SH, Pascual-Leone A, Bikson M. Fundamentals of transcranial electric and magnetic stimulation dose: definition, selection, and reporting practices. Brain Stimul. 2012;5(4):435-53.

33. Pierrot-Deseilligny E, Burke D. The circuitry of the human spinal cord - spinal and corticospinal mechanisms of movement. New York: Cambridge University Press; 2012.

34. Ranck JB Jr. Which elements are excited in electrical stimulation of mammalian central nervous system: a review. Brain Res. 1975;98(3):417-40.

35. Rossini P, Barker A, Berardelli A, Caramia M, Caruso G, Cracco R, et al. Noninvasive electrical and magnetic stimulation of the brain, spinal cord and roots: basic principles and procedures for routine clinical application. Report of an IFCN committee. Electroencephalogr Clin Neurophysiol. 1994;91(2):79-92.

36. Rossini P, Burke D, Chen R, Cohen LG, Daskalakis Z, Di lorio R, et al. Noninvasive electrical and magnetic stimulation of the brain, spinal cord, roots and peripheral nerves: basic principles and procedures for routine clinical and research application. An updated report from an I.F.C.N. committee. Clin Neurophysiol. 2015;126(6):1071-107.

37. Roth BJ. Mechanisms for electrical stimulation of excitable tissue. Crit Rev Biomed Eng. 1994;22(3-4):253-305.

38. Ruffini G, Wendling F, Merlet I, Molaee-Ardekani B, Mekonnen A, Salvador R, et al. Transcranial current brain stimulation (tCS): models and technologies. IEEE Trans Neural Syst Rehabil Eng. 2013;21(3):333-45.

39. Rush S, Abildskov JA, McFee R. Resistivity of body tissues at low frequencies. Circ Res. 1963;12:40-50.

40. Salvador R, Silva S, Basser PJ, Miranda PC. Determining which mechanisms lead to activation in the motor cortex: a modeling study of transcranial magnetic stimulation using realistic stimulus waveforms and sulcal geometry. Clin Neurophysiol. 2011;122(4):748-58.

41. Salvador R, Wenger C, Nitsche MA, Miranda PC. How electrode montage affects transcranial direct current stimulation of the human motor cortex. Conf Proc IEEE Eng Med Biol Soc. 2015;2015:6924-7.

42. Song W, Truong DQ, Bikson M, Martin JH. Transspinal direct current stimulation immediately modifies motor cortex sensorimotor maps. J Neurophysiol. 2015;113(7):2801-11.

43. Standring $\mathrm{S}$, et al. Grey's anatomy: the anatomical basis of clinical practice. 40th ed. London: Churchill Livingston Elsevier; 2008.

44. Struijk JJ, Holsheimer J, van Veen BK, Bomm HB. Epidural spinal cord stimulation: calculation of field potentials with special reference to dorsal column nerve fibers. IEEE Trans Biomed Eng. 1991;38(1):104-10.

45. Struijk JJ, Holsheimer J, van der Heide GG, Boom HB. Recruitment of dorsal column fibers in spinal cord stimulation: influence of collateral branching. IEEE Trans Biomed Eng. 1992;39(9):903-12.
46. Struijk JJ, Holsheimer J, Boom HB. Excitation of dorsal root fibers in spinal cord stimulation: a theoretical study. IEEE Trans Biomed Eng. 1993; 40:632-9.

47. Surowiec A, Stuchly SS, Eidus L, Swarup A. In vitro dielectric properties of human tissues at radiofrequency. Phys Med Biol. 1987;32(5):615-21.

48. Wolters C. Influence of Tissue Conductivity Inhomogeneity and Anisotropy on EEG/MEG based Source Localization in the 6 Human Brain. No. 39 in MPI Series in Cognitive Neuroscience. Leipzig: MPI of Cognitive Neuroscience. 2003. ISBN 3-7 936816-11-5.

49. Zareen N, Shinozaki M, Ryan D, Alexander H, Amer A, Truong DQ, Khadka N, Sarkar A, Naeem S, Bikson M, Martin JH. Motor cortex and spinal cord neuromodulation promote corticospinal tract axonal outgrowth and motor recovery after cervical contusion spinal cord injury. Exp Neurol. 2017;297: 179-89. https://doi.org/10.1016/j.expneurol.2017.08.004. Epub 2017 Aug 10.

\section{Publisher's Note}

Springer Nature remains neutral with regard to jurisdictional claims in published maps and institutional affiliations.

\section{Ready to submit your research? Choose BMC and benefit from:}

- fast, convenient online submission

- thorough peer review by experienced researchers in your field

- rapid publication on acceptance

- support for research data, including large and complex data types

- gold Open Access which fosters wider collaboration and increased citations

- maximum visibility for your research: over $100 \mathrm{M}$ website views per year

At BMC, research is always in progress.

Learn more biomedcentral.com/submissions 